\title{
Towards chemical analysis of nanostructures in biofilms II: tip- enhanced Raman spectroscopy of alginates
}

\author{
Journal Article \\ Author(s): \\ Schmid, Thomas; Messmer, Andreas; Yeo, Boon-Siang; Zhang, Weihua; Zenobi, Renato (1) \\ Publication date: \\ 2008-07 \\ Permanent link: \\ https://doi.org/10.3929/ethz-b-000412096 \\ Rights / license: \\ In Copyright - Non-Commercial Use Permitted \\ Originally published in: \\ Analytical and Bioanalytical Chemistry 391(5), https://doi.org/10.1007/s00216-008-2101-1
}




\title{
Towards chemical analysis of nanostructures in biofilms II: tip-enhanced Raman spectroscopy of alginates
}

\author{
Thomas Schmid • Andreas Messmer • Boon-Siang Yeo • \\ Weihua Zhang $\cdot$ Renato Zenobi
}

Received: 23 December 2007 / Revised: 20 March 2008 / Accepted: 25 March 2008/Published online: 23 April 2008

(C) Springer-Verlag 2008

\begin{abstract}
This study examines the feasibility of using tipenhanced Raman spectroscopy (TERS) for label-free chemical characterization of nanostructures in biological systems. For this purpose, a well-defined model system consisting of calcium alginate fibers is studied. In a companion paper, calcium alginate fibers and their network structures were shown to be a good model for the extracellular polysaccharides of biofilms at the nanoscale. TERS analysis of biological macromolecules, such as alginates, is complicated by heterogeneity in their sequence, molecular weight, and conformations, their small Raman cross-section, and the large number of functional groups, which can chemically interact with the silver surface of the tip and cause significant band shifts. Due to these effects, Raman frequencies in TERS spectra of biopolymers do not necessarily resemble band positions in the normal Raman spectrum of the bulk material, as is the case for less complex samples (e.g., dye molecules) studied so far. Additionally, analyte decomposition due to laser heating can have a significant influence, and carbon contamination signals can sometimes even overwhelm the weak analyte signals. Based on the investigation of alginates, strategies for spectra correction, choice of appropriate reference samples, and data interpretation are presented. With this approach, characteristic frequency ranges and specific marker bands can be found for biological macromolecules that can be employed for their identification in complex environments.
\end{abstract}

T. Schmid · A. Messmer • B.-S. Yeo • W. Zhang • R. Zenobi $(\bowtie)$ Department of Chemistry and Applied Biosciences, ETH Zurich, 8093 Zurich, Switzerland

e-mail: zenobi@org.chem.ethz.ch
Keywords Alginate · Biological samples · Biofilm · Tip-enhanced Raman spectroscopy (TERS) · Atomic force microscopy (AFM)

\section{Introduction}

Alginates are linear, unbranched polysaccharide copolymers containing 1,4-linked $\alpha$-L-guluronic acid and $\beta$-D-mannuronic acid in different amounts and sequences. Their molecular weight can vary over a wide range, with $100 \mathrm{kDa}$ being a typical value [1-3]. At low $\mathrm{pH}$, alginic acid forms a waterinsoluble gel. A similar hydrogel is formed when alginate solutions are mixed with calcium salts. Mainly guluronic acid-rich parts of alginate interact with $\mathrm{Ca}^{2+}$ ions, which can cross-link different alginate strands resulting in a threedimensional network-like structure with water-enclosing pores, that is a stable hydrogel [2-4]. Alginates are found as stabilizing compounds in the extracellular polymeric substances (EPS) of certain biofilms. Commercially available alginates are usually extracted from the cell wall of brown algae. In contrast to algal alginates, bacterial alginates are partially acetylated on the O-2 and O-3 positions of the mannuronate units, which protects them from conversion into guluronate by C-5 epimerase [5]. This is a possible explanation for their guluronate/mannuronate ratio, which is usually lower than in algal alginates.

Algal alginates are employed as a matrix material in cell immobilization, transplantation, and tissue engineering [1, $6,7]$, and as a wound dressing material [8] and excipient in drug delivery systems [4]. Polysaccharide hydrogels, such as alginate gels, are known to have physicochemical properties similar to those of biofilms $[9,10]$. Thus, alginates 
are thought to be a good model system for the investigation of these microbial aggregates [11]. In another study, we have shown that alginate fiber bundles, their network structures and hydrogels are a good model for the architecture of the EPS matrix at the nanometer scale as well [12]. In atomic force microscopy (AFM) images calcium alginate hydrogels and EPS of river-water biofilms have shown structures of 100 - to $400-\mathrm{nm}$ width and 2- to 10 -nm thickness. The investigation of river-water biofilms by a combined confocal laser-scanning microscopy (CLSM)-AFM method demonstrated the heterogeneity of the extracellular matrix at the nanometer scale and the need for a chemical characterization method for such nanostructures [12]. In this study, we investigate the feasibility of using tip-enhanced Raman spectroscopy (TERS) for spatially resolved, label-free chemical characterization of biological systems [13, 14]. Here, calcium alginate acts as a model system for biofilm EPS and biological samples in general, which often are soft materials with low Raman scattering cross-sections.

TERS is derived from surface-enhanced Raman spectroscopy (SERS) and is based on the enhancement of Raman signal intensities by a laser-illuminated, sharp metal or metal-coated tip positioned in the optical near field of the sample (i.e., within a few nanometers above the sample surface). Since the enhancement takes place only on the sample area directly under the nanometer-sized tip, this technique allows Raman mapping with a spatial resolution of approx. 20-50 nm [15-18]. In depth, the enhancement is restricted to approx. 10-20 nm $[18,19]$. In SERS and TERS, the enhancement is generally attributed to two mechanisms: electromagnetic enhancement and chemical enhancement [20]. The latter is also called the charge transfer (CT) mechanism. Electromagnetic enhancement is based on the excitation of surface plasmons by the laser. Surface plasmons can be understood as collective oscillations of free electrons, which propagate along metal surfaces with strong evanescent fields reaching into the surrounding medium. The enhanced electromagnetic field is strongest at the metal surface and decays exponentially over several nanometers from the surface, the so-called near field. In TERS, this effect leads to an enhancement of Raman bands of molecules located in the near field of a resonantly excited metal-coated or metal tip. On the other hand, chemical enhancement can take place when molecules are in direct contact with a TERS tip and interact chemically through their functional groups with the metal surface. The Raman signals of vibrations of these functional groups can be enhanced due to $\mathrm{CT}$ processes between molecule and metal surface and thus an overall greater polarizability. Since the chemical environment of the functional group is changed, the CT mechanism can lead to frequency shifts of the enhanced bands.

TERS has already been applied successfully to the investigation of dye molecules, carbon nanotubes, adenine nanocrystals, and DNA [21]. The first demonstration of TERS on biological nanostructures was its application to the ommatidial lens surfaces of insect eyes [22]; more recently, TERS spectra from bacterial surfaces were obtained using silver-coated AFM tips [23, 24]. Recent demonstrations of detection of cytochrome c [25] and single-stranded RNA [26] show the potential of this technique for identification and localization of important fractions of biological systems, such as proteins and nucleic acids.

This study is based on a well-defined model system for EPS polysaccharides and other biopolymers, with the aim of elucidating and overcoming some difficulties in the chemical analysis of such complex systems. At this stage of our research on biological systems, we do not focus on the spatial resolution that can be achieved by TERS, but on spectroscopic properties of weakly Raman scattering biopolymers, the interpretation of their spectra, and the identification of marker bands that allow their detection in complex matrices. A recent study on the spectroscopic properties of cytochrome $\mathrm{c}$ has revealed significant differences between TER spectra on the one hand and normal Raman and SER spectra on the other hand, which is a consequence of the small volume fraction probed by TERS as compared to the ensemble measurements in normal Raman spectroscopy and SERS [25]. In the case of alginates, we expect analogous effects, which complicate the interpretation of their spectra.

TERS analysis of biological macromolecules, such as alginates, is complicated by heterogeneity in their sequence, molecular weight, and conformations, their small Raman cross-section, and the large number of binding sites, which can chemically interact with the silver surface of the tip in different ways. TERS measurements of adenine have already demonstrated that chemical interactions between nitrogen lone pairs and the tip can induce changes in the TER spectrum [27]. Due to these effects, Raman shifts in TERS spectra of biopolymers do not necessarily resemble band positions in the normal Raman spectrum of the bulk material, as is the case for simple systems (e.g., dye molecules) studied so far [21], because chemical effects due to direct interactions between functional groups and the silver surface can lead to significant band shifts. Since alginates are weak Raman scatterers with a large number of potential binding sites (e.g., a carboxylic acid function in every monomeric subunit), chemical enhancement and related band shifts are expected to play a significant role. Additionally, analyte decomposition due to laser heating can have an influence, and the resulting bands from carbon contaminations can sometimes even overwhelm the weak analyte signals. Based on the present investigation of alginates, strategies for spectra correction, choice of appropriate reference samples, and data interpretation for complex biological samples are presented. 


\section{Experimental}

\section{Apparatus}

A homebuilt combination of three commercial instruments was employed in this study. An AFM (Explorer, Veeco Instruments, Santa Barbara, CA, controlled by SPMLab software, Veeco Instruments), an inverted CLSM (FluoView FV500, Olympus, Melville, NY), and a Raman spectrometer (Holospec f/1.8i, Kaiser Optical Systems, Ann Arbor, MI) equipped with a front-illuminated charge-coupled device (CCD) detector (Andor Technologies, Belfast, UK) that can be used either independently or simultaneously to investigate the same part of the sample [28]. Figure 1a shows the measurement principle of the apparatus. Samples were coated onto transparent glass slides and placed on top of the inverted CLSM. For optical imaging and excitation of Raman signals, a continuous-wave laser beam $(\lambda=532 \mathrm{~nm}$, Ventus, Laser Quantum, Stockport, UK) was coupled into the CLSM and focused onto the sample by a $\times 60 / 1.4$ N.A. oil immersion objective. Based on a comparison of alginate spectra, the 532-nm laser was chosen for these investigations instead of a 488-nm model (Chromalase, Blue Sky Research, Milpitas, CA) used in a former study [12], for two reasons: first, the longer wavelength resulted in a clearly reduced fluorescence background; secondly, the reduction of the Raman intensity due to the $\nu^{4}$ dependence (with $\nu$ denoting the frequency of the laser radiation) was more than compensated by the higher laser power of the 532-nm model (200 $\mu \mathrm{W}$ at the sample compared with $30 \mu \mathrm{W}$ for the $488-\mathrm{nm}$ laser). A folding mirror in the CLSM beam path allows the rapid switching between CLSM imaging and spectroscopic analysis. This mirror allows the light backscattered by the sample to pass out of the microscope, via an edge filter (532 nm, Semrock, Rochester, NY), and is coupled into the Raman spectrograph. The AFM head can be mounted on top of the inverted CLSM and aligned with respect to the CLSM as described previously [12]. For image processing and analysis the software SPIP 4.2 (Image Metrology, Lyngby,

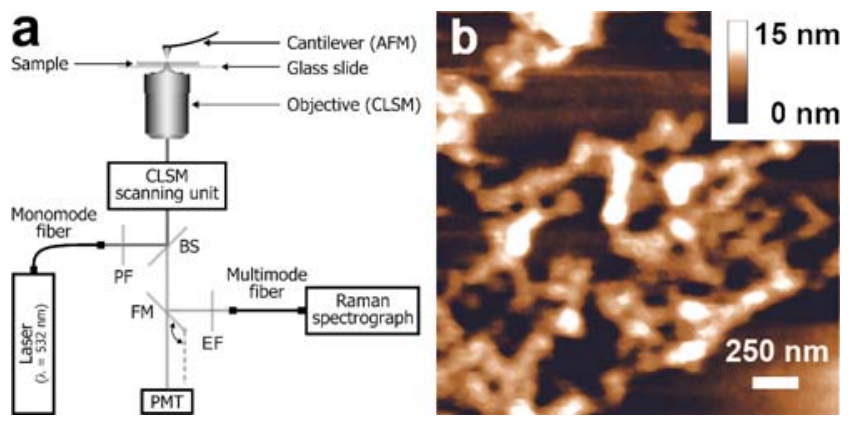

Fig. 1 a Schematic of the instrumental setup (see text for details): $P F$ plasma filter, $B S$ beam splitter, $F M$ folding mirror, $E F$ edge filter, $P M T$ photomultiplier tube. b AFM contact mode topography image of calcium alginate fibers
Denmark) was employed. Except for the background subtraction mentioned in the text, no correction of the Raman spectra, such as baseline subtraction or smoothing, was made. For presentation of the spectra, the software Plot 0.995 for Mac OSX (Michael Wesemann, plot.micw.eu) was used.

\section{AFM probes}

Contact mode AFM imaging was performed with silicon tips having radii of curvature of $<10 \mathrm{~nm}$ (PPP-CONTR from Nanosensors). For TERS measurements, contact mode silicon nitride AFM tips (OMCL RC800PSA, Olympus) were coated with a $30-\mathrm{nm}$ layer of $\mathrm{AlF}_{3}$ and subsequently with a $30-\mathrm{nm}$ layer of $\mathrm{Ag}$ in a vapor coating system (MED020, Baltec, Balzers, Liechtenstein) with a rate of approx. $0.05 \mathrm{~nm} \mathrm{~s}^{-1}$. Other studies by our group have shown that underlayers consisting of a material having a refractive index lower than that of silicon, such as $\mathrm{SiO}_{\mathrm{X}}$ and $\mathrm{AlF}_{3}$, can shift the plasmon resonance frequency of the Ag top layer and lead to higher enhancement factors when 488-nm excitation is employed $[29,30]$. Simulations of localized plasmon resonances have shown that for 532-nm excitation, similar or even higher field enhancements can be expected [31, 32].

\section{Purification of alginate}

For spectroscopic investigations, sodium alginate had to be purified, because commercially available alginates are contaminated with polyphenols and other compounds, which lead to a brownish color of the alginate powder and whose fluorescence emission hampers the collection of Raman spectra. Therefore, a purification procedure similar to that used by Skjakbraek et al. [33] was applied. A $100-\mathrm{mL}$ aliquot of $1 \%$ sodium alginate solution (Lancaster Synthesis, Alfa Aesar, Heysham, UK) was rigorously stirred and heated to $75{ }^{\circ} \mathrm{C}$. Successively, four portions of $250 \mathrm{mg}$ sodium chlorite were added, each of them followed by $250 \mu \mathrm{L}$ glacial acetic acid with a reaction time of 15 min between each addition. The mixture was stirred for one additional hour and the reaction was terminated by cooling in an ice bath and precipitating the alginate by addition of approx. $100 \mathrm{~mL}$ ethanol. The colorless, gel-like precipitate was washed with ethanol and acetone and dried in a desiccator. For further purification, the sample was dissolved again in $100 \mathrm{~mL}$ deionized water and mixed with approx. $100 \mathrm{~mL}$ ethanol under rigorous stirring, yielding a finely dispersed, colorless precipitate that was filtrated over glass wool because conventional membrane filters were clogged by the gel-like material. The product was washed with ethanol and dried in a desiccator. Other strategies for purification of alginate yielded a partially depolymerized product leading to a dramatically reduced viscosity of the corresponding aqueous solutions (bleaching with approx. $20 \% \mathrm{H}_{2} \mathrm{O}_{2}$ ) or 
only to a small reduction of the brown color of the alginate powder (e.g., washing the powder several times in different solvents).

Generation of $\mathrm{Ag}$ colloids inside alginate layers

In order to simulate a silver-coated TERS tip in contact with the sample, alginates were mixed with Ag colloids. When commercially available silver colloids or SERS substrates (glass slides vapor-coated with 6-nm silver) were used, Raman bands where detected that can be assigned to amorphous carbon (data not shown) [34]. This is probably a consequence of decomposition of alginates due to heating or catalytic effects induced by the silver surface. Another explanation for this behavior is carbon contamination (or precursor molecules) already present on the silver surface before adsorption of alginates. Alginates are weak Raman scatterers, i.e., their spectrum can be easily overwhelmed by carbon bands even in the presence of just small amounts of contaminants. Therefore a strategy was developed where silver grains were freshly generated inside alginate samples.

Alginate layers were prepared by drop-coating of $100 \mu \mathrm{L}$ $2 \%$ sodium and calcium alginate, respectively, onto glass slides. Subsequently, the samples were immersed into a $2 \%$ silver nitrate solution overnight in a dark environment. After washing with water to remove excess silver salt, the samples were dried in a desiccator and investigated by CLSM and Raman spectroscopy. In the laser focus, silver grains were generated photochemically inside the alginate samples. Silver generation was also observed when these samples were stored in a bright environment for several hours. According to a previously described mechanism, carboxylic acid functions of alginate are oxidized during irradiation, while $\mathrm{Ag}(\mathrm{I})$ is reduced to $\mathrm{Ag}(0)$ [35]. The oxidized $\mathrm{COO}^{-}$ functions are lost in the form of $\mathrm{CO}_{2}$, and other binding sites of alginate can interact with the freshly prepared silver surface. We suppose that for the case of calcium alginate another mechanism has to be taken into account. Even after washing of the samples, chloride ions were present inside the hydrogel (from $\mathrm{CaCl}_{2}$ ), which led to the formation of a silver chloride precipitate that could be observed in some cases even with the naked eye. Similar to the classical photographic process based on silver bromide, silver chloride decomposes easily into silver and chlorine under the action of light.

\section{Results}

AFM imaging of calcium alginate fibers

The samples investigated in this study by AFM and TERS were well-separated calcium alginate nanostructures on glass slides. Compared with a former study [12], an improved sample preparation method had to be applied, since otherwise the alginates were wiped away by the tip in contact mode AFM measurements. Glass slides were first treated with $\mathrm{Ca}(\mathrm{OH})_{2}$ solution overnight and subsequently stored in $\mathrm{CaCl}_{2}(0.2 \mathrm{M})$ before use, in order to electrostatically immobilize $\mathrm{Ca}^{2+}$ ions on the glass surface, to improve its interaction with the alginate. Prior to spin coating, the glass slides were washed with deionized water and methanol and dried in a stream of nitrogen gas. Subsequently, a diluted (200 $\mathrm{mg} \mathrm{L}^{-1}$ ) sodium alginate solution was spin-coated followed by treatment with $500 \mu \mathrm{L}$ calcium chloride solution $(0.2 \mathrm{M})$ yielding well-separated calcium alginate aggregates. After $5 \mathrm{~min}$, the $\mathrm{CaCl}_{2}$ solution was removed by spin coating, and the slides were air-dried in a dust-free environment for at least $1 \mathrm{~h}$ before investigation. AFM topography images of such samples showed aggregates of 3- to 10-nm-thick calcium alginate fibers (Fig. 1b). Since other AFM studies revealed a single-strand thickness of similar polysaccharides of approx. $1 \mathrm{~nm}$ [36], we interpret our data with alginate fibers that are side-by-side aggregates of only a few alginate molecules, which are cross-linked by calcium ions. In alginate hydrogels and biofilm EPS, such polysaccharide fiber bundles form three-dimensional networks with water-enclosing pores [37].

\section{Raman spectroscopy of alginates}

For collection of reference spectra of bulk alginate without an enhancing tip, $100 \mu \mathrm{L}$ of a $2 \%$ solution of purified alginate was drop-coated onto a cleaned glass slide and allowed to dry, resulting in an alginate layer much thicker than the diameter of the laser focus. Calcium alginate bulk samples were prepared by immersion of alginate-coated glass slides into $10 \mathrm{~mL} 0.2 \mathrm{M} \mathrm{CaCl}_{2}$ solution overnight, washing with water, and air drying. By means of the CLSM objective, a laser beam with a wavelength of $532 \mathrm{~nm}$ and a power of approx. $200 \mu \mathrm{W}$ was focused onto the sample. Spectra 1 and 2 in Fig. 2 represent the average of ten Raman spectra of sodium and calcium alginate, respectively, each of them recorded with a collection time of $60 \mathrm{~s}$. The spectra exhibit a weak, continuous fluorescence background probably due to residual contaminants and distinct Raman bands that can be attributed to alginate.

The most prominent bands and their assignments according to Raman and infrared spectra of alginate and other polysaccharides are summarized in Table 1. The normal Raman spectra of alginates can be divided into two parts: vibrations of the polymer backbone in the range of $<1,300 \mathrm{~cm}^{-1}$ and stretching vibrations of the carboxylate functional groups with Raman shifts at $\geq 1,300 \mathrm{~cm}^{-1}$. Interactions of alginate with calcium ions lead to changes of band positions with the most pronounced band shift of the symmetric $\mathrm{COO}^{-}$ 


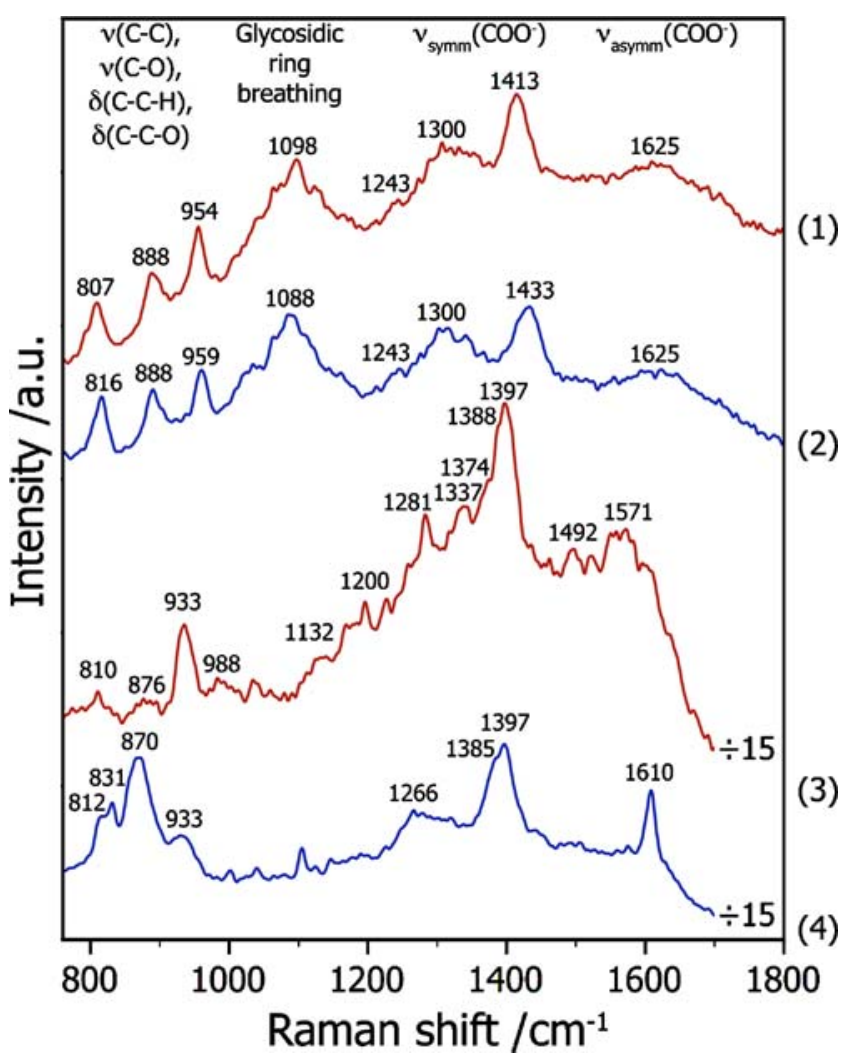

Fig. 2 Raman reference spectra of sodium (1) and calcium alginate bulk samples (2) as well as sodium (3) and calcium alginate samples mixed with silver colloids (4). See text for measurement parameters

stretching band from $1,413 \mathrm{~cm}^{-1}$ (Na alginate) to $1,433 \mathrm{~cm}^{-1}$ (Ca alginate). As previously described in an IR spectroscopy study, band shifts in this range are an indicator for the calcium content in alginate gels [38]. In agreement with these results, dipping of a sodium alginate layer into $\mathrm{CaCl}_{2}$ solution for a few seconds to minutes led to gel formation, but no band shifts were observed in the Raman spectra. The hydrogel was saturated with $\mathrm{Ca}^{2+}$ only after immersion of the alginate into $\mathrm{CaCl}_{2}$ solution for several hours, as indicated by a reproducible band shift of $20 \mathrm{~cm}^{-1}$. This behavior confirms the assignment of this mode to carboxylate groups, which directly interact with the $\mathrm{Ca}^{2+}$ ions and contradicts an assignment of this signal to a $\mathrm{CH}_{2}$ deformation mode in a former Raman study [3]. An empirical method has been previously published that provides insight into the nature of carboxylate bonding in organometallic complexes [39] and was recently applied successfully to interactions between carboxylates and metal surfaces [40]. By investigating the difference between the frequencies of asymmetric and symmetric carboxylate stretching modes, the model allows one to discriminate different species, such as ionic form, monodentate binding of one ligand, bidentate or monodentate bridging of two ligands, and chelation. According to this model, we can assign the small shift of $20 \mathrm{~cm}^{-1}$ to binding of calcium ions via both oxygen atoms of the carboxylate functions (bidentate bridging or chelating). In the range of glycosidic ring breathing modes, shifts to lower wavenumbers (e.g., 1,098 to $1,088 \mathrm{~cm}^{-1}$ ) are generally observed, which were attributed to a weakening of $\mathrm{C}-\mathrm{C}$ and $\mathrm{C}-\mathrm{O}$ bonds as an indirect consequence of the binding of alginate to calcium [38].

We expect this band assignment to also be valid for bacterial alginate. As mentioned in the Introduction, there are two main differences between commercially available algal alginates and the bacterial product: partial acetylation of mannuronate residues and a higher fraction of guluronate units in the latter. The guluronate/mannuronate ratio in alginates has been shown to affect only relative band intensities, but not the frequencies of Raman bands [2, 3]. A Raman study of cellulose has revealed that acetylation

Table 1 Most prominent Raman bands and tentative band assignments for normal Raman spectra and silver colloid-enhanced Raman spectra of sodium alginate and calcium alginate

\begin{tabular}{|c|c|c|c|c|c|}
\hline Band assignment & $\begin{array}{l}\text { Na alginate } \\
\left(\mathrm{cm}^{-1}\right)\end{array}$ & $\begin{array}{l}\text { Ca alginate } \\
\left(\mathrm{cm}^{-1}\right)\end{array}$ & Reference & $\begin{array}{l}\mathrm{Na} \text { alginate }+\mathrm{Ag} \\
\left(\mathrm{cm}^{-1}\right)\end{array}$ & $\begin{array}{l}\mathrm{Ca} \text { alginate }+\mathrm{Ag} \\
\left(\mathrm{cm}^{-1}\right)\end{array}$ \\
\hline \multirow{4}{*}{$\begin{array}{l}\text { Skeletal } \mathrm{C}-\mathrm{C}, \mathrm{C}-\mathrm{O} \text { stretching, and } \mathrm{C}-\mathrm{C}-\mathrm{H} \text {, } \\
\mathrm{C}-\mathrm{C}-\mathrm{O} \text { bending modes }\end{array}$} & 807 & 816 & \multirow[t]{4}{*}[2,3]{} & 810 & 812 \\
\hline & 888 & 888 & & 876 & 831 \\
\hline & \multirow[t]{2}{*}{954} & \multirow[t]{2}{*}{959} & & 933 & 870 \\
\hline & & & & 988 & 933 \\
\hline Glycosidic ring breathing mode & 1,098 & 1,088 & {$[43]$} & 1,132 & 1,104 \\
\hline $\begin{array}{l}\text { Carboxylate stretching vibration: symmetric stretching } \\
\text { or } \mathrm{C}-\mathrm{O} \text { single bond stretching vibration }\end{array}$ & $1,300^{1}$ & 1,300 & {$[38-40,44]$} & 1,281 & 1,266 \\
\hline \multirow[t]{4}{*}{ Symmetric carboxylate stretching vibration } & \multirow[t]{4}{*}{1,413} & \multirow[t]{4}{*}{1,433} & \multirow[t]{4}{*}[38-40,44]{} & 1,337 & \\
\hline & & & & 1,374 & \\
\hline & & & & 1,388 & 1,385 \\
\hline & & & & 1,397 & 1,397 \\
\hline \multirow[t]{2}{*}{ Asymmetric carboxylate stretching vibration } & \multirow[t]{2}{*}{1,625} & \multirow[t]{2}{*}{1,625} & \multirow{2}{*}[38-40,44]{} & 1,492 & 1,610 \\
\hline & & & & 1,571 & \\
\hline
\end{tabular}


also does not affect band positions assigned to the polysaccharide backbone [41]. There were only weak additional bands observed, which can be assigned to the acetyl groups. Therefore, we can expect to find the same Raman frequencies from the alginate backbone and a few additional bands, when significantly acetylated bacterial alginate is analyzed.

Influence of silver colloids on the Raman spectrum of alginates

In order to simulate a TERS tip in contact with alginates and to investigate the influence of the silver surface on their spectra, silver colloids were generated inside alginate bulk samples as described in the Experimental. It has been shown for adenine that significant band shifts of 10 $20 \mathrm{~cm}^{-1}$ are obtained and even new bands can appear in TERS spectra compared with normal Raman spectra depending on which nitrogen atoms interact chemically with the silver-coated tip [27]. For alginate, a polymer with many different binding sites, an even stronger influence of the silver surface is expected. Light from the 532-nm laser with a power of approx. $200 \mu \mathrm{W}$ was focused onto different parts of the samples and Raman spectra were collected with an acquisition time of $30 \mathrm{~s}$. Very intense spectra such as traces 3 and 4 in Fig. 2 were obtained from sodium and calcium alginate, respectively, in a reproducible way. For a better comparability with the normal Raman spectra 1 and 2, the intensity of the colloid-enhanced spectra 3 and 4 in Fig. 2 was scaled by a factor of $1 / 15$. Compared with the normal Raman spectra, band shifts were observed in the presence of Ag colloids, which indicate direct, chemical interactions between alginate and silver and are discussed below.

\section{TERS of alginates}

TERS measurements were performed on well-separated, nanometer-sized aggregates of calcium alginate fibers on a glass slide as shown in Fig. 1b. Confocal images of the sample - as revealed by the insets in Fig. 3-were recorded, and $\mathrm{AlF}_{3} / \mathrm{Ag}$-coated $\mathrm{AFM}$ tips were approached to alginate aggregates. Subsequently, the 532-nm laser beam was focused on the tip apex by means of a $\times 60 / 1.4$ N.A. objective, and TER spectra were recorded with a collection time of $30 \mathrm{~s}$. In order to reduce sample decomposition, the laser power was lowered to $30 \mu \mathrm{W}$. Spectrum 1 in Fig. 3 is a typical result of such an experiment.

With the tip retracted, a far-field spectrum was collected on the same spot of the sample as shown in Fig. 3, trace 3. Even with a 10-min collection time and the same laser power, no Raman bands could be identified in the spectra as a consequence of the small amount of sample material in the laser focus. The TERS data with the tip in contact with the sample are shown as trace 1 . In the range of $<1,100 \mathrm{~cm}^{-1}$

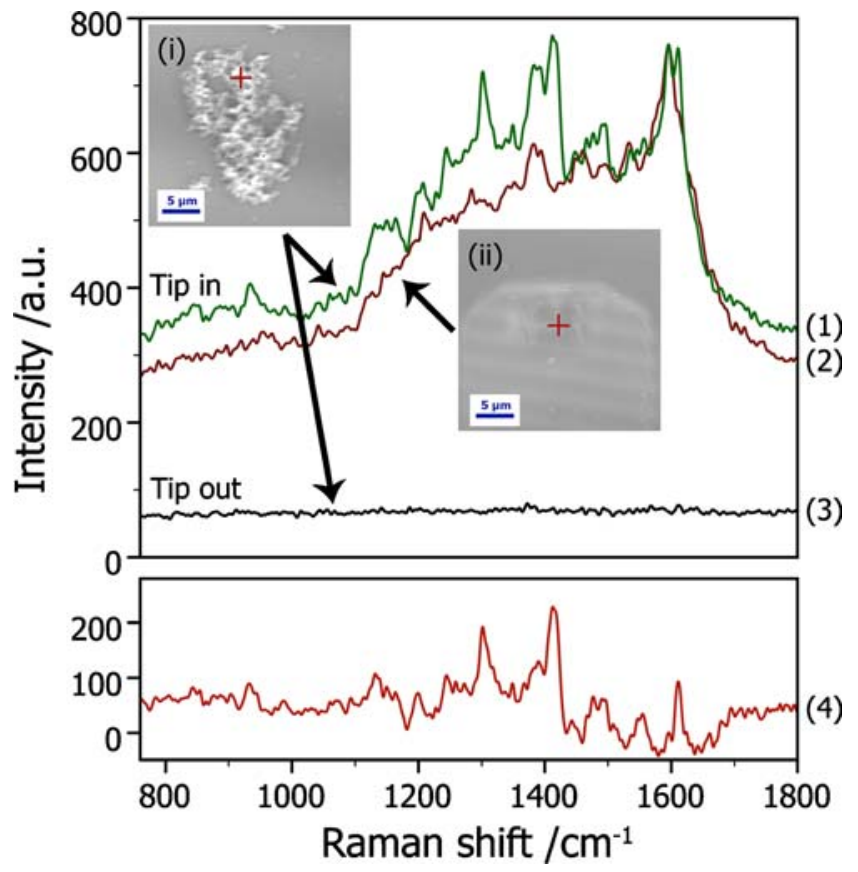

Fig. 3 TERS raw data of calcium alginate fibers and subtraction of a carbon contamination spectrum: 1 near-field alginate TER spectrum, 2 spectrum of the carbon contamination on the tip, 3 far-field spectrum of alginate collected with the tip retracted, and 4 'carbon-corrected' difference between spectra 1 and 2 . Insets $i$ and $i i$ reveal confocal microscopy images of the parts of the sample where spectra 1-3 were collected

only a few bands were present in the TERS spectra, including the band at $933 \mathrm{~cm}^{-1}$ which was found in every TER spectrum of alginate measured in this study. Based on the $933-\mathrm{cm}^{-1}$ band, the TERS enhancement factor was estimated. There are two quantities that can be used to gauge the TERS enhancement: the observed enhancement (OE), also termed as 'optical contrast' or just 'contrast', and the net enhancement (NE) [21]. OE is the ratio between nearfield $\left(I_{\mathrm{NF}}\right)$ and far-field $\left(I_{\mathrm{FF}}\right)$ signal intensities. In a TERS experiment, $\mathrm{OE}$ is usually determined by measuring signal intensities with the tip in contact with the sample $\left(I_{\text {tip in }}\right)$ and with the tip retracted $\left(I_{\text {tip out }}\right)$ :

$\mathrm{OE}=\frac{I_{\mathrm{NF}}}{I_{\mathrm{FF}}}=\frac{I_{\text {tip in }}-I_{\text {tip out }}}{I_{\text {tip out }}}=\frac{I_{\text {tip in }}}{I_{\text {tip out }}}-1$

$\mathrm{NE}$ additionally takes into account that the far-field and near-field signals arise from different volume fractions of the sample. Whereas the far-field spectrum stems from the sample inside the whole laser focus, the near-field signal is generated only in the much smaller near field around the tip. As shown by the AFM image in Fig. 1b, the thickness of the sample is in the range of $10-20 \mathrm{~nm}$, similar to the near field around the tip, i.e., this defines the thickness of the volume giving rise to both near-field and far-field signals in this case. Since the thickness of both volumes is 
the same, only the different areas, $A_{\mathrm{FF}}$ and $A_{\mathrm{NF}}$, have to be taken into account:

$\mathrm{NE}=\mathrm{OE} \frac{A_{\mathrm{FF}}}{A_{\mathrm{NF}}}$

Even though no signals could be detected in the far-field measurements, a rough estimation of the enhancement factor for the $933-\mathrm{cm}^{-1}$ band can be given. For the calculation of the $\mathrm{OE}$, the peak intensity in the near-field spectrum was compared with an estimated maximum value of the far-field signal. For this estimation, the standard deviation of the noise in the far-field spectrum was calculated in the range of this band (i.e., $912-965 \mathrm{~cm}^{-1}$ ). According to the IUPAC definition of the limit of detection (LOD), the upper limit for the far-field signal was calculated as three times the standard deviation of the noise. The signal/noise ratio of the $933-\mathrm{cm}^{-1}$ band is approximately 20 . The calculation of the enhancement factor takes into account that also with 20 times longer accumulation time, no far-field signals were detected. Based on Eqs. (1) and (2) and parameters already used in Ref. [30], an observed enhancement (OE) of approx. $\times 100$ and a net enhancement (NE) of $\geq 2 \times 10^{4}$ were obtained, which are in good agreement with TERS data for other molecules [21, 30].

More and much stronger bands were observed between 1,100 and $1,700 \mathrm{~cm}^{-1}$, a range that is known to be influenced by amorphous carbon contaminations in SERS and TERS experiments [34]. Elemental and amorphous carbon are strong Raman scatterers, whose bands are resonantly enhanced in the UV, visible, and NIR range [42]. Thus, even small amounts of contaminants adsorbed to the tip can have a significant influence on the TERS spectrum of weak Raman scatterers, such as alginates. For short collection times (tenths of seconds) the spectrum of amorphous carbon consists of sharp bands, which fluctuate in Raman shift and intensity, representing different carbon modifications and average out with long collection times $(>10 \mathrm{~s})$ to give the two broad bands centered at approx. 1,360 and $1,590 \mathrm{~cm}^{-1}$ [34]. In the case of alginate TERS spectra, the bands between 1,100 and $1,700 \mathrm{~cm}^{-1}$ are surely not only due to amorphous carbon, for two reasons: first, even with a long acquisition time of $30 \mathrm{~s}$ sharp bands are observed; and second, the band positions do not jump when repeated measurements were performed on the same spot of the sample (see below). Rather, we interpret these features as a superposition of alginate and carbon signals as demonstrated by spectrum 2 in Fig. 3, which was recorded by placing the tip on a blank part of the sample after using it for four alginate measurements. The average of three repeated measurements reveals a spectrum of amorphous carbon with the two broad bands mentioned above. Figure 3 demonstrates that the sharp bands in the range of 1,100 $1,700 \mathrm{~cm}^{-1}$ are located on top of a broad background that can be ascribed to carbon contaminations adsorbed to the tip. Since the carbon contamination was observed from the first measurement with every tip and remained at an approximately constant level over several measurements, the main source for carbon contaminations is in this case not sample decomposition, but carbon contamination (or precursor molecules) already deposited during or directly after the vapor coating process. In contrast to TERS measurements of other molecules (e.g., dye molecules [21]), the carbon contamination signals dominate the spectra due to the small Raman cross-section of the alginate analyte molecules. In order to facilitate the interpretation of the TERS spectra, the carbon signals were normalized to the intensity of the strongest peak at approx. $1,590 \mathrm{~cm}^{-1}$ and subtracted as a background. Only 'carbon-corrected' spectra, such as spectrum 4 in Fig. 3 were further analyzed. Here, mainly the range $<1,550 \mathrm{~cm}^{-1}$ was taken into account, because the subtraction procedure led to artifacts in some spectra in the form of negative peaks just below the strongest band at $1,590 \mathrm{~cm}^{-1}$ due to imperfect fitting.

Figure 4 shows TERS spectra (after background subtraction) that were collected with three different tips on different spots of a calcium alginate fiber sample: Spectra 1-3 are repeat measurements on the same spot of the sample, spectra

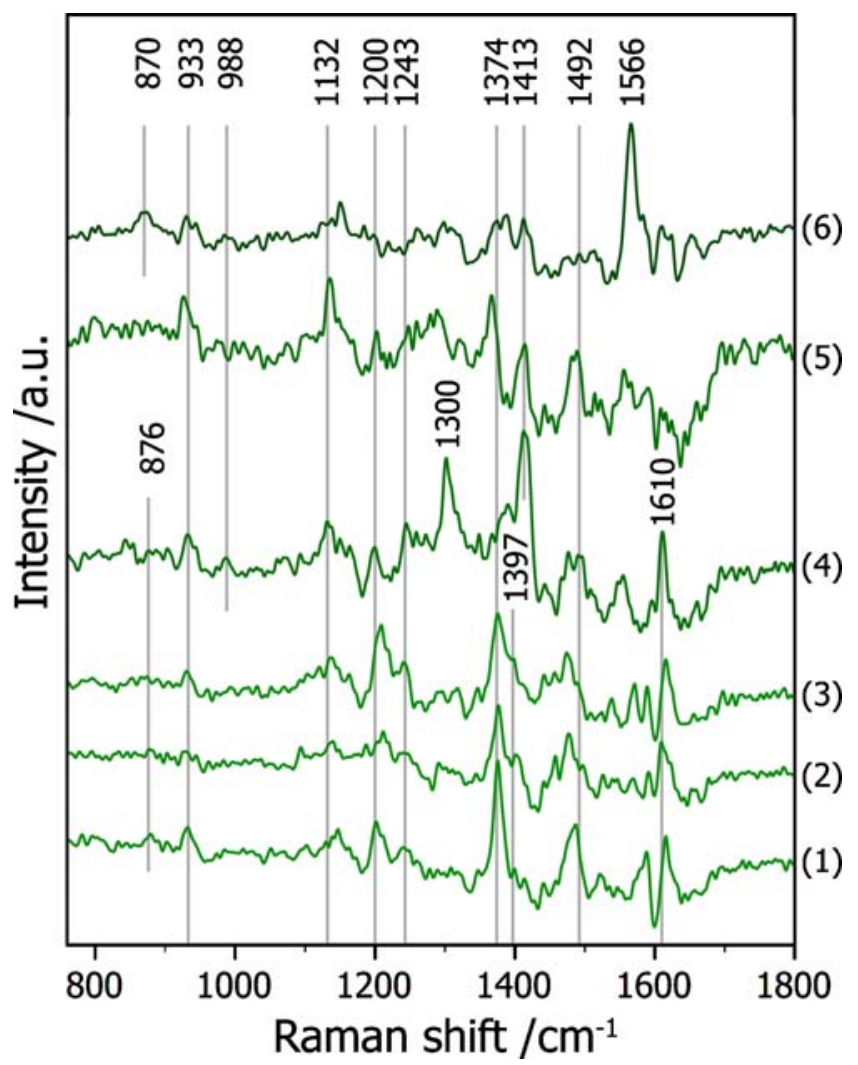

Fig. 4 Background-corrected TERS spectra of calcium alginate fiber bundles collected with three different tips: spectra $1-3$ with tip 1 on the same spot of the sample, spectra 4 and 5 with tip 2 on 2 different spots of the sample, and spectrum 6 with tip 3 
4 and 5 are recorded with a different tip on two different alginate clusters, and spectrum 6 was measured with a third $\mathrm{AlF}_{3} / \mathrm{Ag}$-coated tip. Several bands are reproducible with different tips and on different sample spots and are in agreement with bands in the reference spectra 3 and 4 in Fig. 2. For other bands, frequency shifts were observed when different tips were placed on different sample spots, and in general there are discrepancies between normal Raman, colloid-enhanced Raman, and TER spectra, which are discussed below.

\section{Discussion}

Band shifts induced by Ag colloids

As mentioned above, Ag colloids in alginate bulk samples induce reproducible frequency shifts in the Raman spectra that can be explained by chemical interactions between functional groups of alginate and the silver surface. The presence of silver caused shifts to lower wavenumbers in the carboxylate range, indicating direct interactions between these functional groups and the metal colloids. The sodium alginate spectrum (Fig. 2, trace 3) shows a superposition of many bands in this range, indicating a variety of interactions between carboxylate groups and silver. This was confirmed by repeated Raman measurements with shorter acquisition times (e.g., $3 \mathrm{~s}$ ), which resulted in fluctuations of the relative intensities of the bands in this range, in some cases leading to distinct bands clearly sticking out (data not shown). The fluctuations can be explained by dynamics in the interaction between different carboxylate binding sites and the silver surface, and therefore enhancement of different carboxylate modes. The calcium alginate spectrum (Fig. 2, trace 4) contains only a few bands in the carboxylate stretching range, indicating fewer possibilities for the carboxylate-silver interaction. This can be explained by blocking of many binding sites by calcium. Guluronic acidrich parts of alginates in particular are known to bind calcium ions very efficiently $[2,3]$, so that mainly mannuronic acidrich substructures can interact with silver.

Almost no enhancement was observed in the range $1,000-1,200 \mathrm{~cm}^{-1}$ in both spectra, which contains the glycosidic ring breathing modes and thus signals from the molecular backbone. Only a shoulder at $1,132 \mathrm{~cm}^{-1}$ and a few weak bands are present in the sodium and calcium alginate spectra, respectively. This confirms that chemical enhancement of signals from functional groups that directly interact with the silver plays a significant role. In the fingerprint range below $1,000 \mathrm{~cm}^{-1}$, enhancement and frequency shifts for all three characteristic alginate bands were observed. This can be explained by interactions between oxygen and silver, which affect $\mathrm{C}-\mathrm{O}$ stretching and $\mathrm{C}-\mathrm{C}-\mathrm{O}$ bending modes found in this range. The band at $933 \mathrm{~cm}^{-1}$ was found to be a good marker band for alginates, because it is present in all spectra of alginates that were in contact with silver, irrespective of counterion or binding state to silver. A tentative assignment of the shifted bands and a comparison with the band positions in the spectra of pure alginates is given in Table 1.

\section{Interpretation of TER spectra}

Several bands in the TER spectra in Fig. 4 are clearly reproducible, even when different tips are used and different spots on the sample are probed. One of them is the marker band at $933 \mathrm{~cm}^{-1}$, which was just mentioned. Different Raman shifts in the carboxylate stretching range are found in the spectra representing different interactions between carboxylate functions and $\mathrm{Ag}$ as well as mixtures of pure ionic alginate and $\mathrm{Ca}$-complexing alginate. The latter depends on which parts of alginate - guluronate-rich (Cabinding), mannuronate-rich, or mixed parts-are in contact with the enhancing sites of the tip. Spectra 1-3 demonstrate

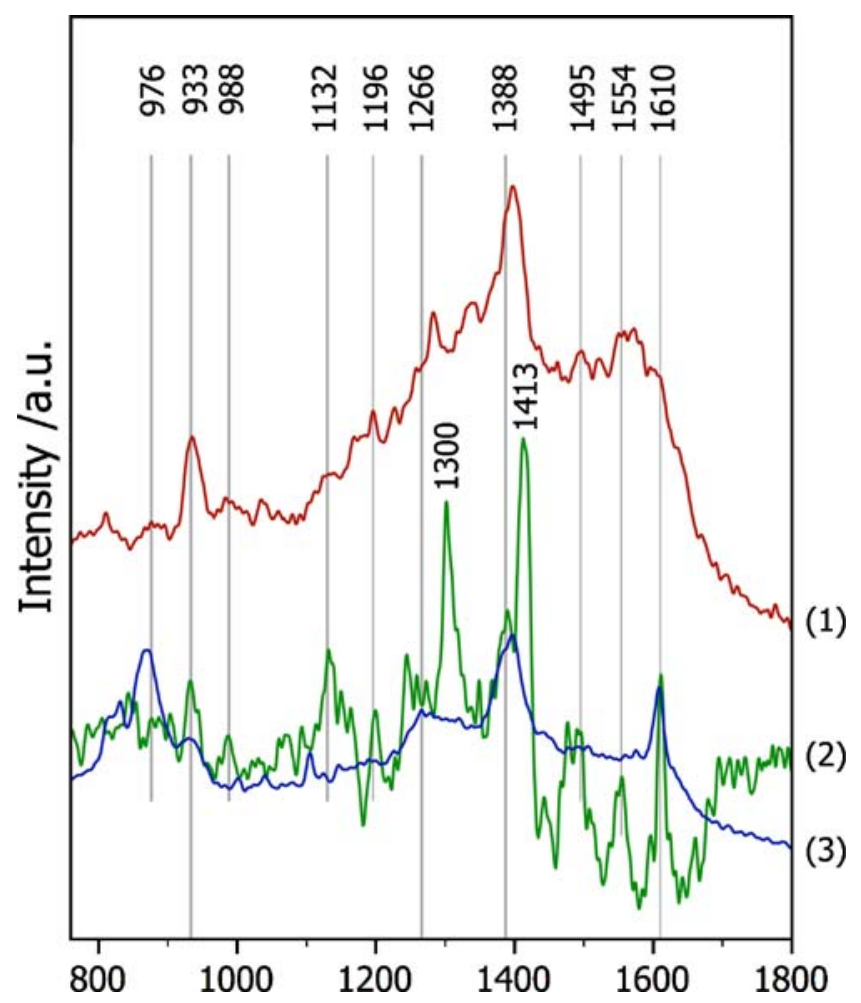

Fig. 5 Comparison of a TERS spectrum 2 (see Fig. 4, trace 4) with reference spectra of sodium (1) and calcium alginate bulk samples mixed with Ag colloids (3). The TERS spectrum can be interpreted as a superposition of the calcium alginate spectrum with carboxylate stretching modes present in the sodium alginate spectrum. The strong bands at $1,300 \mathrm{~cm}^{-1}$ and $1,413 \mathrm{~cm}^{-1}$ represent symmetric carboxylate stretching vibrations found in the spectrum of sodium alginate without Ag (see Fig. 2, trace 1), which can be explained by electromagnetic enhancement of an ionic alginate species 
the good reproducibility of the spectra when the same part of the sample is probed. When other positions are analyzed (with different tips), changes in the carboxylate stretching range are observed, whereas for the bands in the fingerprint range $<1,100 \mathrm{~cm}^{-1}$ - especially for the $933-\mathrm{cm}^{-1}$ marker band-high reproducibility was achieved. In the carboxylate stretching range, clear differences between the colloidenhanced spectra in Fig. 2 traces 3 and 4 and the TER spectra are observed, since the first is a bulk measurement, whereas in the latter only a small fraction of alginate is probed by the TERS tip. The discrepancy in the probed volume fractions can lead to significant differences in the Raman spectra of biopolymers. This effect has already been observed in a recent TERS study on cytochrome $\mathrm{c}$ by our group [25], where only in TER spectra the weak signature of aromatic amino acids could be identified, whereas in normal Raman and SERS, the spectrum was overwhelmed by the strong resonant Raman scattering of heme. Only by probing a very small fraction of a cytochrome c layer was there a chance of probing the apoprotein part of the molecules with the TERS tip and selectively enhancing the amino acid bands. In the case of alginate, many different alginate-silver interactions are probed simultaneously in colloid-enhanced measurements resulting in a broad overlap of different bands, whereas only few different interactions are seen in the TER spectra, leading to relatively sharp and distinct bands in the carboxylate range.

Most of the labeled bands in Fig. 4 can be found in the spectra of sodium or calcium alginate that is in contact with Ag colloids (spectra 3 and 4 in Fig. 2). An exception is spectrum 4 in Fig. 4, where additional bands at 1,300 and $1,413 \mathrm{~cm}^{-1}$ are strongly enhanced. As revealed by Fig. 5, this TERS spectrum (here spectrum 2) can be understood as a superposition of the 'calcium alginate $+\mathrm{Ag}$ ' reference spectrum (spectrum 3) and some carboxylate bands from the 'sodium alginate $+\mathrm{Ag}$ ' spectrum (spectrum 1) representing different chemical interactions between carboxylate groups and $\mathrm{Ag}$. The strong bands at $1,300 \mathrm{~cm}^{-1}$ and $1,413 \mathrm{~cm}^{-1}$ resemble bands of the pure sodium alginate (without Ag, see Fig. 2, trace 1) and can be explained by electromagnetic enhancement of a free ionic species of alginate by the $\mathrm{Ag}$ nanostructures without band shifts induced by chemical effects.

\section{Conclusions}

The first application of TERS on alginates has shown the possibility to enhance and collect spectra of these weak Raman scatterers in order to identify them inside a biological matrix. The investigation of these well-defined models for real biological systems has demonstrated some basic differences between biological macromolecules and more simple systems, such as dye molecules, studied so far by TERS [21]. Due to the heterogeneity in sequence, conformation, complexation of counterions, and molecular weight as well as the large number of different interaction sites between analyte and tip, the TERS spectrum of a macromolecule does not necessarily resemble the band positions of the Raman spectrum of the bulk material. Chemical enhancement and related band shifts are expected to play a significant role mainly in the investigation of macromolecules with low Raman scattering cross-sections. Additionally, the interpretation of the spectra can be complicated by signals of amorphous carbon contaminations on the tip. Even small amounts of contaminants can have a significant influence on the spectrum of weak Raman scatterers. We have shown that these issues can be solved by background subtraction and by careful selection of reference materials, taking into account chemical effects caused by interactions between functional groups of the analyte and the silver surface of the tip. With these strategies, characteristic frequency ranges and specific marker bands can be found for biological macromolecues that can be applied for their identification in complex biological systems. The goal of further studies is to improve TERS towards a robust tool for the analysis of biological samples, which allows, for example, the elucidation of the distribution of different biopolymers (e.g., polysaccharides, proteins, and nucleic acids) inside the biofilm matrix and can be applied in many other fields of medicine and biology.

Acknowledgements Financial support for our work from the Deutsche Forschungsgemeinschaft (to Thomas Schmid), the ETH Zürich, and the Gebert Rüf Stiftung (grant no. P-085/03) is greatly appreciated.

\section{References}

1. Martinsen A, Skjakbraek G, Smidsrod O (1989) Biotechnol Bioeng 33:79-89

2. Chourpa I, Carpentier P, Maingault P, Dubois P (1999) Proc SPIE 3608:48-54

3. Chourpa I, Carpentier P, Maingault P, Fetissoff F, Dubois P (2000) Proc SPIE 3918:166-173

4. Tonnesen HH, Karlsen J (2002) Drug Dev Ind Pharm 28:621-630

5. Windhues T, Borchard W (2003) Carbohydr Polym 52:47-52

6. Rowley JA, Madlambayan G, Mooney DJ (1999) Biomaterials 20:45-53

7. Smidsrod O, Skjakbraek G (1990) Trends Biotechnol 8:71-78

8. Paul W, Sharma CP (2004) Trends Biomater Artif Organs 18:18-23

9. Tielen P, Strathmann M, Jaeger KE, Flemming HC, Wingender J (2005) Microbiol Res 160:165-176

10. Strathmann M, Griebe T, Flemming HC (2000) Appl Microbiol Biot 54:231-237

11. Wloka M, Rehage H, Flemming HC, Wingender J (2004) Colloid Polym Sci 282:1067-1076

12. Schmid T, Burkhard J, Yeo BS, Zhang W, Zenobi R (2008) Anal Bioanal Chem. DOI 10.1007/s00216-008-2100-2

13. Stöckle RM, Suh YD, Deckert V, Zenobi R (2000) Chem Phys Lett 318:131-136 
14. Anderson MS (2000) Appl Phys Lett 76:3130-3132

15. Hayazawa N, Yano T, Watanabe H, Inouye Y, Kawata S (2003) Chem Phys Lett 376:174-180

16. Hartschuh A, Anderson N, Novotny L (2003) J Microsc-Oxford 210:234-240

17. Pettinger B, Ren B, Picardi G, Schuster R, Ertl G (2005) J Raman Spectrose 36:541-550

18. Mehtani D, Lee N, Hartschuh RD, Kisliuk A, Foster MD, Sokolov AP, Maguire JF (2005) J Raman Spectrosc 36:1068-1075

19. Hartschuh A, Sanchez EJ, Xie XS, Novotny L (2003) Phys Rev Lett 90:095503

20. Hering K, Cialla D, Ackermann K, Dörfer T, Möller R, Schneidewind H, Mattheis R, Fritzsche W, Rösch P, Popp J (2008) Anal Bioanal Chem 390:113-124

21. Schmid T, Yeo BS, Zhang W, Zenobi R (2007) Use of tipenhanced vibrational spectroscopy for analytical applications in chemistry, biology, and materials science. In: Kawata S, Shalaev V (eds) Tip enhancement. Elsevier, Amsterdam

22. Anderson MS, Gaimari SD (2003) J Struct Biol 142:364-368

23. Neugebauer U, Rosch P, Schmitt M, Popp J, Julien C, Rasmussen A, Budich C, Deckert V (2006) Chem Phys Chem 7:1428-1430

24. Neugebauer U, Schmid U, Baumann K, Ziebuhr W, Kozitskaya S, Deckert V, Schmitt M, Popp J (2007) Chem Phys Chem 8:124137

25. Yeo BS, Mädler S, Schmid T, Zhang W, Zenobi R (2008) J Phys Chem C 112:4867-4873

26. Bailo E, Deckert V (2008) Angew Chem Int Edit 47:1658-1661

27. Hayazawa N, Watanabe H, Saito Y, Kawata S (2006) J Chem Phys 125:
28. Vannier C, Yeo BS, Melanson J, Zenobi R (2006) Rev Sci Instrum 77:023104

29. Yeo BS, Zhang W, Vannier C, Zenobi R (2006) Appl Spectrosc 60:1142-1147

30. Yeo BS, Schmid T, Zhang W, Zenobi R (2007) Anal Bioanal Chem 387:2655-2662

31. Cui X, Zhang W, Yeo BS, Zenobi R, Hafner C, Erni D (2007) Opt Express 15:8309-8316

32. Zhang W, Cui XD, Yeo BS, Schmid T, Hafner C, Zenobi R (2007) Nano Lett 7:1401-1405

33. Skjakbraek G, Murano E, Paoletti S (1989) Biotechnol Bioeng 33:90-94

34. Kudelski A, Pettinger B (2000) Chem Phys Lett 321:356-362

35. Yonezawa Y, Sato T, Ohno M, Hada H (1987) J Chem Soc Faraday T I 83:1559-1567

36. McIntire TM, Brant DA (1997) Imaging carbohydrate polymers with noncontact mode atomic force microscopy. In: Townsend RR, Hotchkiss AT (eds) Techniques in glycobiology. Marcel Dekker, New York

37. Decho AW (1999) Carbohydr Res 315:330-333

38. Sartori C, Finch DS, Ralph B, Gilding K (1997) Polymer 38:43-51

39. Deacon GB, Phillips RJ (1980) Coord Chem Rev 33:227-250

40. Yeo BS, Chen ZH, Sim WS (2003) Langmuir 19:2787-2794

41. Adebajo MO, Frost RL, Kloprogge JT, Kokot S (2006) Spectrochim Acta A 64:448-453

42. Ferrari AC, Robertson J (2001) Phys Rev B 64:075414

43. Schenzel K, Fischer S (2001) Cellulose 8:49-57

44. Ribeiro CC, Barrias CC, Barbosa MA (2004) Biomaterials $25: 4363-4373$ 